\title{
Anti-sunlight Jamming Technology of Laser Fuze
}

\author{
Xiangjin Zhang ${ }^{1, *}$, Ruoyu $\mathrm{Wu}^{1}$, Chengfang Shen ${ }^{1}$ and Wei Dai ${ }^{2}$ \\ ${ }^{1}$ ZNDY of Ministerial Key Laboratory, Nanjing University of Science and Technology, Nanjing 210094, China \\ ${ }^{2} 63963$ army, China \\ ${ }^{*}$ Corresponding author
}

\begin{abstract}
Environmental elements, especially sunny weather, can easily interfere with the operation of a pulsed laser fuze. The solar radiation entering the detection system causes the fuze to produce a "false alarm." Consequently, the bomb may not work accurately. On the basis of the traditional theory of spectral radiation and the detection principle, this study establishes a transmission model of the solar radiation with loss medium damage to analyze the characteristics of sunlight interference. This work studies and simulates anti-sunlight methods, from the receipt of echo signals entering the receiving system to the reduction of the sunlight entering the system. Finally, on the basis of the small impact of laser detection systems, this study builds a test platform to verify the anti-sunlight measures.
\end{abstract}

Keywords-laser fuze; anti-sunlight; detection technology

\section{INTRODUCTION}

A pulsed laser fuze is a laser detection system whose operation requires high accuracy and is affected by unavoidable external disturbances. The sun, as the largest source of radiation, covers a wide range of wavelengths [1,2]. The spectral response range of several common laser detectors at present is within this range of wavelength coverage. When sunlight enters the receiving field and is received with the target echo signal, the judgment of the target echo signal by the system is affected and the detection capability of the system is seriously reduced. Conventional ammunition is currently widely used, but its general size is small and the occupancy of the fuze is restricted. Meanwhile, the components of conventional ammunition are often loaded with tens of thousands of $\mathrm{g}$ because of the limit of their launching environment. Currently, the solar radiation interference of laser imaging systems and space borne laser detection systems and alarm technologies have been studied in depth [3]. The research on the anti-sunlight interference of ammunitions in low-load emission environments, such as missiles, has developed but is not suitable for conventional ammunition in low-load environments. Reducing or eliminating sunlight interference has become a primary research subject related to improving the detection performance of the miniaturized impact laser near fry fuze. According to the research requirements of the pulsed laser fuze against sunlight interference, this work establishes a transmission model that is based on classical optical radiation theory for the corresponding carrier and proposes schemes for resisting sunlight interference. Experimental results from simulation and verification tests are also provided.

\section{ANALYSIS OF CHARACTERISTICS OF SUNLIGHT INTERFERENCE}

According to relevant tests, strong laser detection systems in environments with solar radiation are prone to false alarms and unstable detection distance. Therefore, a solar radiation transmission model needs to be established, and the characteristics of sun interference have to be analyzed. This study establishes an atmospheric transmittance model for miniaturized impact laser detection systems to address the uncertainty of target location and the influence of environmental factors by combining the advantages of the calculation method of two atmospheric transmittances [4], an empirical formula, and table lookup.

Factors affecting atmospheric transmittance include atmospheric absorption and scattering. According to Lambert Bill's law, the expression of atmospheric transmittance is as follows:

$$
\tau_{d}(\lambda)=\tau_{s}(\lambda) \tau_{a}(\lambda)
$$

$\tau_{a}(\lambda), \tau_{s}(\lambda)$ indicate the transmittances associated with absorption and scattering, respectively, and $\tau_{d}(\lambda)$ is a function of wavelength lambda $\lambda$, atmospheric thickness, and medium density $\rho$.

In this study, a pulsed laser with a detection band of 905 $\mathrm{nm}$ is used, and the spectral response range of the typical photo detector is 400-1100 $\mathrm{nm}$. The main absorption component in the atmosphere is water vapor, and the absorption of carbon dioxide and ozone is not apparent to the aforementioned band[5]. When atmospheric transmittance is calculated, the absorption of water vapor by the solar radiation is mainly considered, and the atmospheric transmittance formula can be simplified as

$$
\tau_{a}(\lambda)=\tau_{a_{\mathrm{H}_{2} \mathrm{O}}}(\lambda) \tau_{s}(\lambda)
$$

The absorption of water vapor is generally expressed as precipitation $\omega$ and expressed as

$$
w=10^{-3} H_{r} H_{a} / d
$$


By using the engineering water vapor spectral transmittance formula proposed by Leckner et al. in McClatchey [5],

$$
\tau_{a}(\lambda)=\tau_{a_{\mathrm{H}_{2} \mathrm{O}}}(\lambda)=\exp \left[-0.2358 k_{a_{\mathrm{H}_{2} \mathrm{O}}} \cdot w \cdot \frac{m_{r}}{\left(1+20.27 k_{a_{\mathrm{H}_{2} \mathrm{O}}} \cdot w \cdot m_{r}\right)^{0.45}}\right]
$$

$k_{a_{H_{2} \mathrm{O}}}$ is the spectral absorption coefficient of water vapor. This coefficient can be found in the table [5]. $m_{r}$ is the relative mass of the atmosphere at standard atmospheric pressure. In a cloudless atmosphere, atmospheric scattering is mainly Rayleigh scattering of solar radiation in air. Spectral transmittance is expressed by using the coefficient proposed by Leckner for the scattering of air to the solar radiation in the atmosphere in response to the Rayleigh scattering of atmospheric air molecules.

$$
\tau_{y \lambda}=\exp \left(-0.00835 \lambda^{-4.08}\right) m_{a}
$$

Solar spectral radiance is expressed according to Planck's radiation law and the definition of radiation emittance and by using the earth orbit eccentricity correction factor $g$ by Daffie and Backman [6] as

$$
L\left(\lambda T_{s}\right)=M\left(\lambda T_{s}\right) \cdot g=\frac{c_{1}}{\lambda^{5}} \frac{1}{e^{\frac{c_{2}}{\lambda T_{s}}}-1} \cdot\left(1+0.033 \cos \frac{2 \pi n}{365}\right)
$$

A laser detector is coaxial with the projectile and the surface of the detector changes with the change in the spatial attitude of the projectile. Conventional artillery, especially for such low-extension ballistic guns as tank guns, is not vertically incident to the surface of the laser detection and receiving optical system. To thoroughly study the interference of sunlight to the laser detection system and the subsequent antiinterference technology, the solar radiation power model of the tilting surface is established. In this study, the solar radiation transmission model is created at a given time, and the inclined sun radiation is as shown in Figure I.

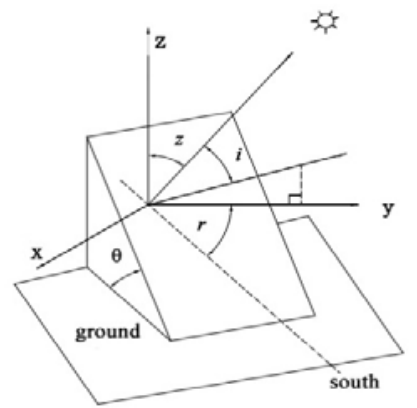

FIGURE I. SOLAR RADIATION ON INCLINED PLANE

In Figure I, $\theta$ is the angle between the inclined and horizontal planes of the detecting device, $h$ is the sun height angle, $\mathrm{r}$ is the azimuth of the slope, and $\mathrm{z}$ is the zenith angle.

The expression of the incident angle $i$ of the sun [7] is

$$
\cos i=\cos \theta \sinh +\sin \theta \cosh \cos (a-r)
$$

According to Formulas (6) and (7), the expression of the solar direct spectral irradiance on the inclined plane $E_{\lambda}(\theta, r)$ is

$$
E_{\lambda}(\theta, r)=\varepsilon_{\lambda} L\left(\lambda T_{s}\right) \tau_{d}(\lambda) \Delta \Omega \cos i=\frac{\pi \varepsilon_{\lambda} L\left(\lambda T_{s}\right) \tau_{d}(\lambda) R_{s}^{2} \cos i}{R_{s e}^{2}}
$$

$\Delta \Omega$ denotes the stereoscopic angle of the sun relative to the earth.

The expression of the full-band solar radiation illuminance on the inclined plane is

$$
E(\theta, r)=\int_{0}^{\infty}\left[E_{\lambda}(\theta, r)\right] d \lambda
$$

This research focuses on studies related to the maximum influence time of solar radiation on the receiving system during summer afternoons.

Forward detection is generally used for laser detection systems in conventional ammunition. Direct, reflected, and scattered sunlight enter the receiving field. The photo detector produces the random fluctuating noise voltage through photoelectric conversion, thereby decreasing the signal-tonoise ratio (SNR) of the detection system. Given the basic characteristics of sun interference, the filter and the adjustment of the bandwidth of the receiving circuit are considered to improve the SNR of the system to achieve the effect of antiinterference.

\section{SimULATION OF ANTI-SUNLIGHT INTERFERENCE}

The model of the space position of the detection target and the typical detection device are established as shown in Figure II.

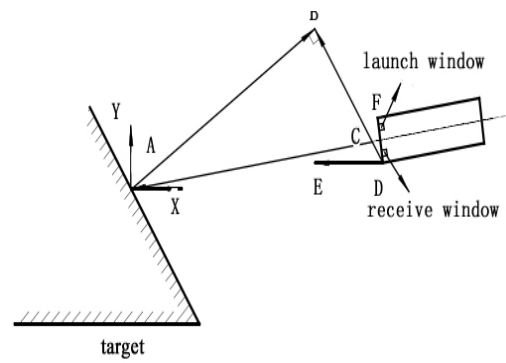

FIGURE II. DETECTING DEVICE AND SPACE POSITION OF DETECTION TARGET

$\angle E D F=\theta$ is the angle between the surface of the detector and the horizontal plane. $\angle B A C=\beta$ is the angle between the optical axis of the detection device and the surface of the detection target. $\angle B D E=\gamma$ is the angle between the target surface and the horizontal plane. The relationship among the three is denoted as $\beta+\gamma=\theta$. 
The typical parameters of the system are as follows: laser power $P_{t}=75 \mathrm{~W}$, photosensitive diameter $d=5 \mathrm{~mm}$ emission efficiency of optical system $k_{e}=0.8$, optical receiving system efficiency $k_{r}=\tau_{t}$, receiver aperture optical system $A_{r}=2 \times 10^{-4} \mathrm{~m}^{2}$, target reflectivity $\rho=0.3$, atmospheric attenuation coefficient at a given distance from laser $\alpha(r)=0.1956, \lambda=905 \mathrm{~nm}$, quantum efficiency $\eta=0.9$, average quantum efficiency $\bar{\eta}=0.58$, and inclination of target surface $\beta=68^{\circ}$.

By using the typical expression of echo power $[8,9]$, the following is obtained:

$$
P_{r}=\frac{P_{t} K_{e} K_{r} A_{r} \rho \varepsilon \gamma \cos \beta}{\pi S^{2}} e^{-2 \alpha \gamma}
$$

\section{A. Simulation of Filter Light Interference Suppression}

Under intense sunlight, the echo energy of the system decreases with the increase in detection distance and the SNR of the system cannot meet the detection requirements. To suppress the solar radiation entering the receiving field of view, a certain measure of spectral filtering must be implemented.

A spectral filter is used to restrain the solar radiation energy that enters the receiving system. A $905 \mathrm{~nm}$ narrowband filter with bandwidths of $100,80,60,40$, and $20 \mathrm{~nm}$ is obtained for numerical simulation. The number of sunlight photons entering the receiving system is shown in Figure III.

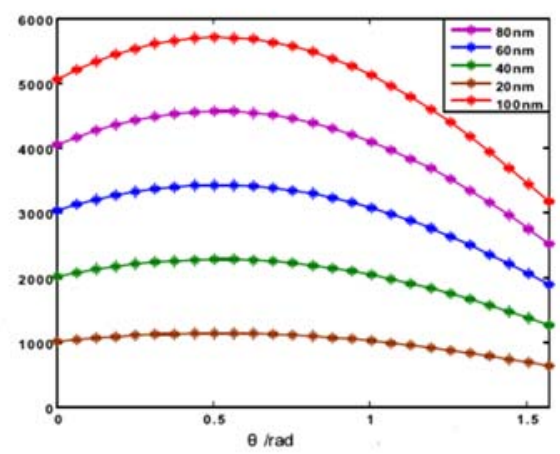

FIGURE III. FILTERING EFFECT OF DIFFERENT BANDWIDTH FILTERS

After the filter is added to the spectrum detection system, the solar radiation entering the receiving system can be effectively suppressed. The narrower the bandwidth of the filter, the better the filter effect. In the suppression of the filter bandwidth of $20 \mathrm{~nm}$, the number of solar radiation entering the sub-receiving system is only approximately 1000 . Therefore, the selected spectrum filter is suitable for solar radiation suppression and produces a good effect.

\section{B. Simulation of Circuit Bandwidth Interference Suppression}

Limiting the bandwidth of the receiving system reduces the amplitude of the target echo signal, shortens the rising time, and decreases the SNR of the system. Meanwhile, limiting the bandwidth of the system can effectively reduce the equivalent noise of the receiving system and improve the SNR. Therefore, an appropriate bandwidth should be selected for the receiving circuit to suppress sunlight interference.

The bandwidth of the system directly affects the mean square value of the circuit noise. Figure IV shows the effect of the receiving system bandwidth on the SNR at various detection distances.

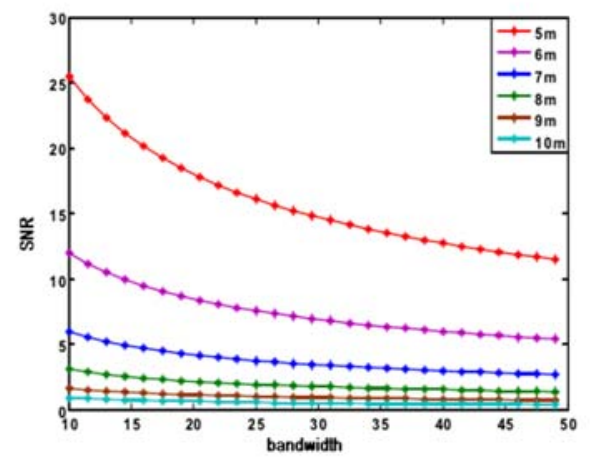

\section{FIGURE IV. RELATIONSHIP CURVES BETWEEN SYSTEM EQUIVALENT BANDWIDTH AND SYSTEM SNR}

When the detection distance is certain, the bandwidth has a great influence on the receiving system. The smaller the bandwidth, the higher the SNR. Furthermore, the bandwidth has a nonlinear relationship with the detection distance. However, when the system bandwidth is too narrow, the receiving system does not match the echo pulse signal. Consequently, the echo signal is distorted and the performance of the detection system is affected. Therefore, selecting the appropriate bandwidth of the receiving system plays an important role in laser fuze operation against sunlight interference and signal reception.

\section{EXPERIMENT}

\section{A. Spectral Filtering Experiment}

On the basis of the small-impact laser detection system, an acquisition experiment of the echo waveform of the detection target is conducted. The waveform acquisition device adopts a Tektronix MD04106-6 oscilloscope; the sampling rate is 5 $\mathrm{GS} / \mathrm{s}$, and bandwidth is $1 \mathrm{GHz}$. The system uses a pulse laser emission frequency of $3 \mathrm{KHz}$, and the gain of the receiving system is approximately $69 \mathrm{~dB}$.

The noise size of the system without sunlight radiation is measured. Then, the collected echo signals are introduced into the data processing software, and the root mean square is calculated. The size of the system noise without sunlight is measured; the weak light in the room is negligible relative to the sunlight. An outdoor test platform is then constructed for the outdoor verification experiment of sunlight interference suppression.

The sunlight irradiance shown by the sun power meter is $1235 \mathrm{~W} \cdot \mathrm{m}^{-2}$ under cloudless outdoor conditions (without considering the tiny floating). The receiving system does not 
add spectral filters. A data acquisition device is used to collect echo signal waveforms from different detection distances. These data are imported into a data processing software to obtain the waveforms shown in Figure V.

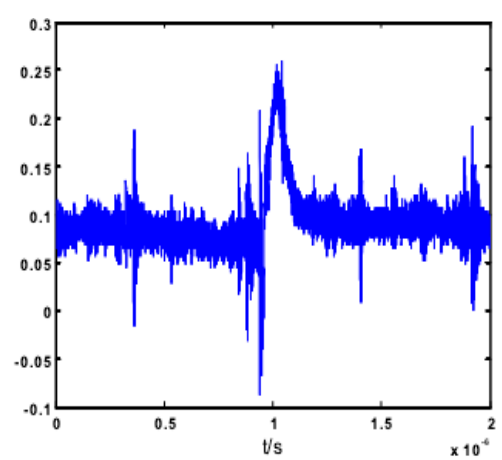

(A) $\mathrm{S}=8 \mathrm{M}$

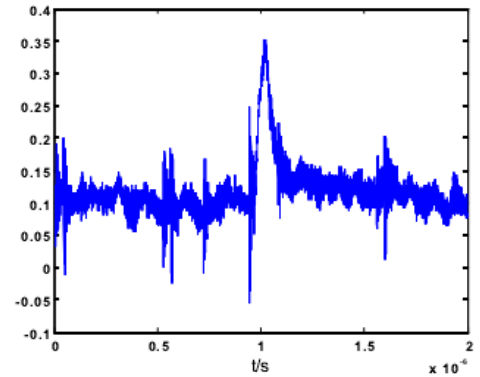

(B) $\mathrm{S}=7 \mathrm{M}$

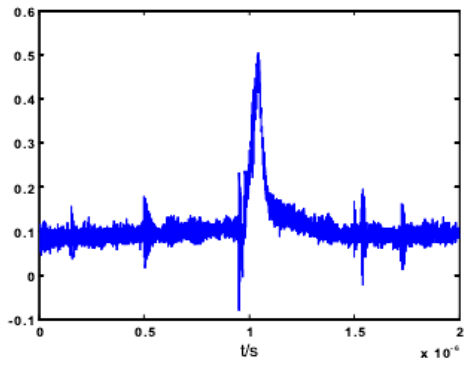

(A) $\mathrm{S}=6 \mathrm{M}$

FIGURE V. WAVEFORMS OF TARGET ECHO SIGNAL WITHOUT FILTER AT DIFFERENT DETECTION DISTANCES

The echo signal is undulating, and a partial noise signal is processed. The root mean square of the system noise is 51.7 $\mathrm{mV}$. The SNRs at detection distances of 6,7 , and $8 \mathrm{~m}$ are approximately $9,6.3$, and 4.5 , respectively.

When the test environment and system parameters are constant, the irradiance of the sun is $1201 \mathrm{~W} \cdot \mathrm{m}^{-2}$. The echo waveforms of the detection target at distances of 6,7 , and $8 \mathrm{~m}$ with the $905 \mathrm{~nm}$ filter are shown in Figure VI.

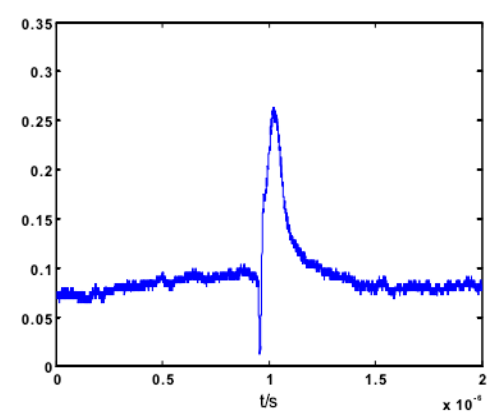

(A) $\mathrm{S}=8 \mathrm{M}$

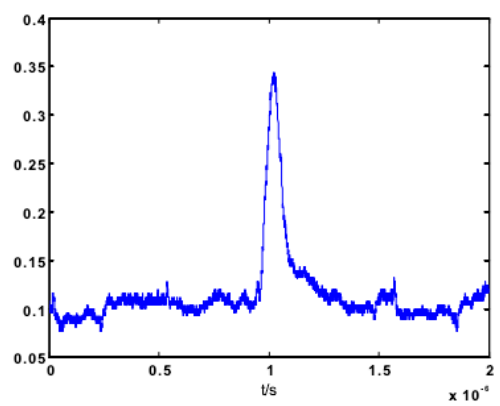

(B) $\mathrm{S}=7 \mathrm{M}$

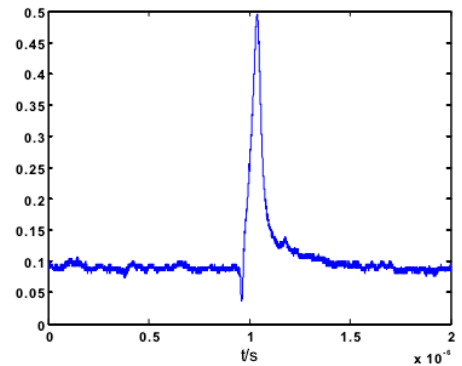

(C) $\mathrm{S}=6 \mathrm{M}$

FIGURE VI. WAVEFORMS OF TARGET ECHO SIGNAL WITH FILTER AT DIFFERENT DETECTION DISTANCES

According to the abovementioned method, the system SNRs at distances of 6,7 , and $8 \mathrm{~m}$ are approximately $31,21.8$, and 15, respectively. Thus, the SNR of the system is noticeably improved. These experimental results show that adding the matching spectral filter to the front end of the detector can largely reduce the influence of the external solar radiation on the echo signal and improve the SNR of the receiving system.

\section{B. Circuit Bandwidth Experiment}

The bandwidth of the receiving circuit of the laser detection system directly affects the SNR.

The effect of sunlight interference is verified under the same environment and the same system parameters by setting the equivalent bandwidth $\Delta \mathrm{f}$ of different circuit by using the oscilloscope of the experimental platform to acquire the noise signal size with solar radiation in the background. The circuit 
bandwidth used in this work is established by the bandwidth selection of the oscilloscope.

Figure VII shows the waveforms collected under the system bandwidths of 60,40 , and $20 \mathrm{MHz}$.

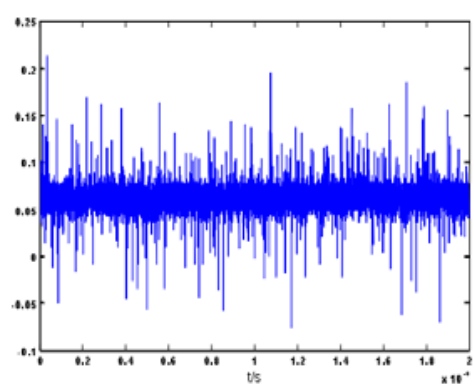

(A) $\Delta f=60 \mathrm{MHz}$

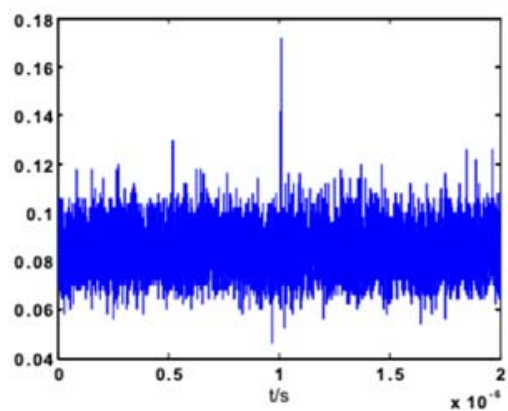

(B) $\Delta f=40 \mathrm{MHz}$

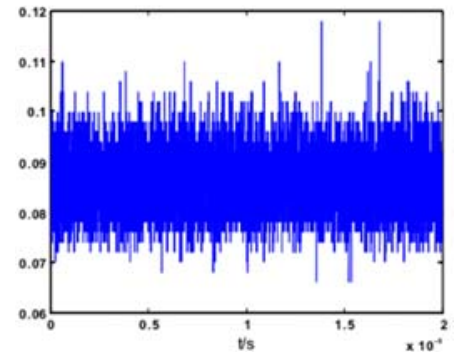

(C) $\Delta f=20 \mathrm{MHz}$

\section{FIGURE VII. WAVEFORMS COLLECTED UNDER DIFFERENT} BANDWIDTHS

The root mean squares of the noise are 53,25 , and $15 \mathrm{mV}$ under receiving system bandwidths of 60,40 , and $20 \mathrm{MHz}$, respectively. Therefore, selecting the appropriate system bandwidth has a certain effect on the suppression of sunlight interference. When designing the receiving circuit, a reasonable selection of circuit bandwidth can effectively restrain the system noise caused by solar radiation and other interferences and improve the detection accuracy of the system.

\section{$\mathrm{V} \quad$ CONCLUSION}

This study explores the operation of the laser fuze while overcoming solar radiation interference using interference suppression theory and experimental research about improving system detection accuracy and reducing the false alarm phenomenon. Moreover, inhibition technology theory is applied to simulate the laser detection of sunlight interference from the echo signals entering the receiving system to inhibit solar radiation. Results show that the spectral filter suppresses sunlight interference well and selecting the appropriate system bandwidth reduces the solar radiation caused by the noise to a certain extent. Furthermore, the use of a small laser detection system to build a test platform for the verification of the sunlight interference suppression effect serves as reference for further research on anti-sunlight interference systems for the laser fuze. For the condition with no sun disturbances, this system can also work correctly because the solution listed in this passage will not influence the transmission of laser. However, such environment is ideal. Actually when the sun's interference is small, it is often accompanied by clouds and rain. These background climates can have a significant impact on laser detection, which is not discussed in detail here.

\section{ACKNOWLEDGEMENT}

This research was financially supported by the National Defense Program Foundation of Science and Technology, China (Grant No. 0106001) and the Weapons and equipment Pre-Research Project of China (Grant No. 41419050202).

\section{REFERENCES}

[1] Wei Weidong, Sun Xiaoquan, Li Honghin, Zhang Yujun. Correlation detection of dual-wavelength laser pulse suppressing background light interference [J].Infrared and laser Engineering,2012, 41(7):1777-1783.

[2] Wang P Y. Beam-shaping optics deliver high-power beams[J]. Laser Focus World, 2001,37(12):115-118.

[3] Zhou G, Liu X, Xu J. A math model of calculate the atmospheric transmittance of infrared radiation[J]. Infrared Technology, 2008, 30(6):331-334

[4] Ping W H. Research into Theoretical Calculation Method on Engineering of Transmittance of Infrared Radiation Through Atmosphere[J]. Optics \& Precision Engineering, 1998.

[5] Bo L. The spectral distribution of solar radiation at the earth's surfaceelements of a model[J]. Solar Energy, 1978, 20(2):143-150.

[6] J. Duffie and W. Beckman, Solar Engineering of Thermal Processes, Wiley, New York, NY, USA, 1980

[7] Cornelius J. Willers. Electro-Optical System Analysis and Design: A Radiometry Perspective[M].2013.

[8] Xu Xiaobin, Zhang He. Optimal Pulsed Frequency and Scanning Speed of Laser Circumferential Detection System[J]. Chinese Laser, 2016(5):195-201.

[9] Zhanghe. Detection And Identification Technology[M].Beijing Institute of Technology press. 